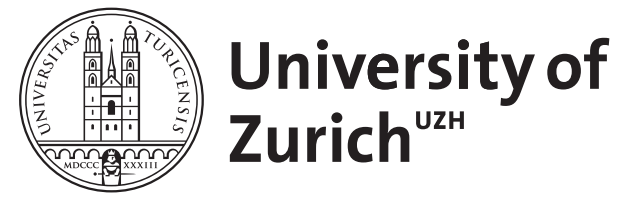

Zurich Open Repository and Archive

University of Zurich

University Library

Strickhofstrasse 39

CH-8057 Zurich

www.zora.uzh.ch

Year: 2008

\title{
Moores Paradox, Behaupten, Urteilen
}

Pfisterer, Christoph C

DOI: https://doi.org/10.1515/cpt-2008-9104

Posted at the Zurich Open Repository and Archive, University of Zurich

ZORA URL: https://doi.org/10.5167/uzh-7358

Journal Article

Published Version

Originally published at:

Pfisterer, Christoph C (2008). Moores Paradox, Behaupten, Urteilen. Conceptus, 37(91):41-62.

DOI: https://doi.org/10.1515/cpt-2008-9104 


\title{
Moores Paradox, Behaupten, Urteilen
}

\author{
Christoph C. Pfisterer
}

\begin{abstract}
Zusammenfassung: Moore ist aufgefallen, dass es absurd ist, Sätze der Form „p, aber ich glaube es nicht" $\mathrm{zu}$ behaupten. Weil es nicht minder widersinnig ist, zu glauben, was ein solcher Satz aussagt, kümmern sich viele Erklärungen für Moores Paradox vorrangig um Überzeugungen. Shoemakers Zurückführung solcher Überzeugungen auf konfligierende Überzeugungen höherer Stufe und Kriegels Diagnose eines logischen Widerspruchs setzen beide fälschlicherweise voraus, dass Behauptungen lediglich geäußerte Urteile sind. Ich plädiere dafür, dass es der episodische Aspekt von Urteilen ist, der es unmöglich macht, eine solche Überzeugung zu haben.
\end{abstract}

Summary: Moore was first to notice that it is absurd to assert sentences of the form "p, but I don't believe it." As it looks even more absurd to believe what such a sentence states, explanations of Moore's paradox have primarily focused on the beliefs thus asserted. Shoemaker, for example, analyzes these beliefs in terms of conflicting higher order beliefs. Kriegel, in return, provides an explanation in terms of logical contradictions. I shall argue that both accounts rest on the mistaken assumption that assertions are merely uttered judgements. What I show instead is that the episodic aspect of judgements renders it impossible even to have such beliefs.

1. G.E. Moore macht uns darauf aufmerksam, dass es doch „perfectly absurd“ ist, einen Satz wie „es regnet, aber ich glaube es nicht" zu äußern, obwohl das, was mit dem Satz ausgesagt wird, "perfectly possible logically“ ist (Moore 1942: 543). In einem Vortrag vor dem Cambridge Moral Science Club im Jahr 1944 berichtet Moore von seiner Entdeckung; das Manuskript 
zu diesem Vortrag ist leider verschollen. Wittgenstein, der sich unter den Zuhörern befand, greift Moores Beobachtung in dem, was als Teil II der Philosophischen Untersuchungen und als Bemerkungen über die Philosophie der Psychologie veröffentlicht ist, auf und verleiht ihr den Namen Moore's Paradox. Gegenüber Malcolm soll Wittgenstein angeblich geäußert haben, dass ihn diese Entdeckung als einzige in Moores Arbeiten wirklich beeindruckt hat (Malcolm 1984: 56). In einer Tagebuchnotiz finden wir folgende Bemerkung: „Moore poked into a philosophical wasp nest with his paradox; \& if the wasps did not duly fly out, that's only because they were to listless" (Wittgenstein 1980: 76). Inzwischen sind die Wespen längst ausgeflogen - die Literatur zu Moores Paradox ist so umfangreich geworden, dass es schwer fallen kann, die philosophisch interessante Problemstellung zu erkennen. ${ }^{1}$ Streitbar scheint vor allem die Frage zu sein, wie Moores Paradox mit dem Sprechakt des Behauptens und dem Fällen von Urteilen zusammenhängt. Ich bin der Ansicht, dass Moores Paradox auf einen wesentlichen Zug von Behauptungen aufmerksam macht, und dass dieser Befund für das Verständnis dessen, was es heißt, ein Urteil zu fällen, fruchtbar gemacht werden kann.

Moores Überlegungen finden sich an verschiedenen Stellen verstreut und man sucht vergeblich nach einer erschöpfenden Darstellung der Paradoxie. Zudem muss man zwischen zwei verschiedenen Paradoxien unterscheiden, die Moore und Wittgenstein nicht immer auseinander halten:

(1) p, aber ich glaube nicht, dass p.

(2) p, aber ich glaube, dass nicht-p.

Sätze beider Typen scheinen einen wahrheitsfähigen propositionalen Gehalt auszudrücken, den man jedoch nicht aufrichtig

${ }^{1}$ Einen Überblick über das Spektrum der Beiträge der letzten sechzig Jahre bietet die Einführung von Green und Williams zu dem kürzlich erschienenen Sammelband zu Moores Paradox (Green/Williams 2007). 
und mit Bedacht in einer Behauptung ausdrücken kann, ohne sich in eine Art Widerspruch zu verstricken. Ich werde mich auf das erste Paradox konzentrieren und zu klären versuchen, worin dieser Widerspruch besteht. Es wird sich herausstellen, dass Sätze dieser Form nicht nur qua Behauptungen defekt sind, sondern einen propositionalen Gehalt ausdrücken, der in einem gewissen Sinn nicht Gegenstand einer Überzeugung sein kann. $^{2}$ Wenn der Nachweis gelingt, dass Sätze im Stil von (1) bereits auf der Ebene des propositionalen Gehalts eine Anomalie aufweisen, dann müssen jene Analysen der Paradoxie eine Lücke hinterlassen, die sich ausschließlich mit dem Behaupten solcher Sätze beschäftigen. Verschiedene Autoren haben auf diesen Mangel von sprechakttheoretischen Analysen hingewiesen und Moores Paradox in Bezug auf Urteile oder bewusste Überzeugungen untersucht (Baldwin 1990, Heal 1994, Rosenthal 1995, Shoemaker 1996, Kriegel 2004). Ich werde dafür argumentieren, dass es richtig ist, Moores Paradox auch in Bezug auf Urteile und Überzeugungen zu analysieren. Im laxen Sinn eines ,inneren Pendants" zu Behauptungen können Urteile Überzeugungen gleichgesetzt werden, da beide eine ähnliche Anomalie aufweisen wie Behauptungen im Stil von (1). ${ }^{3}$ Damit ist jedoch die Gefahr verbunden, das Paradox in Bezug auf Behauptungen als ein

${ }^{2}$ Der Ausdruck „Gegenstand einer Überzeugung“ ist irreführend, da er nahe legt, dass Überzeugungen von propositionalen Gehalten handeln. Wer jedoch von etwas überzeugt ist, ist nicht von einem Gehalt überzeugt, sondern davon, dass sich eine Sache so und so verhält. Die hier behandelten Autoren, die Moores Paradox in Bezug auf Überzeugungen untersuchen, scheinen von den Schwierigkeiten dieses Sprachgebrauchs nicht zurückzuschrecken und ,believing the propositional content“ (Shoemaker 1996: 75) bzw. „,believing those contents" (Kriegel 2004: 114) für eine geeignete Ausdrucksweise zu halten.

3 Die Nachlässigkeit dieser Gleichsetzung verschleiert wichtige Unterschiede zwischen Urteilen und Überzeugungen; so weist „Urteil" bspw. eine Akt-Objekt-Ambiguität auf, über die der Ausdruck „Überzeugung“ nicht verfügt. Ich werde später auf diesen Unterschied eingehen. 
davon abgeleitetes Problem zu verstehen. Wer den Standpunkt vertritt, dass eine Behauptung im Stil von (1) ihre Anomalie vom propositionalen Gehalt einer entsprechenden Überzeugung erbt, setzt implizit voraus, dass Behauptungen nichts anderes als sprachlich veräußerlichte Überzeugungen sind. Am Beispiel von Shoemaker und Kriegel wird gezeigt, dass dieser Standpunkt mit einem falschen Verständnis von Behauptungen operiert und Behauptungen lediglich die Rolle eines Nexus für Behauptungen beimisst. Dieser Fehler lässt sich vermeiden, wenn man, einer Idee von Dummett folgend, den umgekehrten Weg beschreitet und Behauptungen nicht als Veräußerlichungen von Urteilen behandelt, sondern als Verinnerlichung von Behauptungen. Ich gehe davon aus, dass Moores Paradox keiner Lösung, sondern einer Auflösung bedarf; d.h. es muss erklärt werden, weshalb Sätze dieser Form weder gedacht noch behauptet werden können. Moores Paradox weist einen für das Denken und Behaupten wesentlichen Aspekt auf und es wäre daher falsch, diesem Paradox mit einer Lösung begegnen zu wollen.

2. Worin besteht die Paradoxie? Erstens sollten wir festhalten, dass ein Satz wie „Es regnet, aber ich glaube es nicht" keinen formalen Widerspruch aufweist. Wer diesen Satz äußert, widerspricht sich nicht; es wird sich zeigen, dass Situationen denkbar sind, in denen sich Sätze dieser Form sogar sinnvoll äußern lassen. Dennoch weist ein solcher Satz etwas Ähnliches wie einen Widerspruch auf (vgl. Wittgenstein 1974: 177). Wenn sich in (1) etwas einem formalen Widerspruch Ähnliches verstecken soll, dann kommen für dieses zweifelhafte Verhältnis nur die beiden Konjunkte in Frage:

(4) Ich glaube nicht, dass $\mathrm{p}$

Es ist jedoch keineswegs offenkundig, wie die Bestandteile (3) und (4) zu einem Widerspruch führen sollen. "Glauben“ 
ist ein opakes Verbum und nicht wahrheitsfunktional. Aus der Tatsache, dass jemand nicht glaubt, dass p, folgt (leider oder glücklicherweise) weder die Wahrheit von $\neg$ p noch die von p. Wenn sich aus (4) jedoch nicht die Wahrheit von $\neg$ p ergibt, dann wird sich (1) auch nicht im Sinne eines formalen Widerspruchs zurückweisen lassen.

Zweitens kann in (1) auch kein materialer Widerspruch im Sinne einer Unverträglichkeit mit der Wahrheit versteckt sein. Schließlich werden wir nicht selten von der Realität eines Besseren belehrt und müssen Teile dessen, was wir glauben, auf Grund neuerer Erkenntnis aufgeben. Es ist ein wesentliches Merkmal von Überzeugungen, dass sie falsch sein können. Dagegen ließe sich einwenden, dass Überzeugungen zwar falsch sein können, dass man aber nicht wie in (1) eine Überzeugung zum Ausdruck bringen kann, die man gleichzeitig in Zweifel zieht. Dieser Gedanke scheint auch Moore vorgeschwebt zu sein, wenn er das Zustandekommen der Paradoxie dadurch erklärt, dass wir mit der Behauptung, dass $\mathrm{p}$ in gewisser Weise implizieren, dass wir auch der Überzeugung sind, dass p:

It seems to me to be nothing mysterious about this sense of 'imply', in which if you assert that you went to the pictures last Tuesday, you imply, though you don't assert, that you believe or know that you did [...]. 'I went to the pictures last Tuesday, but I don't believe that I did' is a perfectly absurd thing to say, although what is asserted is something which is perfectly possible logically: it is perfectly possible that you did go to the pictures and yet you don't believe that you did [...]. But nevertheless your saying that you did, does imply (in another sense) that you believe you did" (Moore 1942: 542-3).

Aus einer Behauptung folgt zwar nicht logisch, dass das Behauptete wahr ist, aber eine Behauptung impliziert, dass derjenige, der die Behauptung aufstellt, von der Wahrheit seiner Behauptung überzeugt ist oder sich zumindest bewusst ist, dass er 
mit der aufgestellten Behauptung vorgibt, von deren Wahrheit überzeugt zu sein. „I think that the sense of 'imply' in question is similar to that in which, when a man asserts anything which might be true or false, he implies that he himself, at the time of speaking, believes or knows the thing in question - a sense in which he implies this, even if he is lying" (ibid.: 541). Dieser Analyse zufolge enthält die Behauptung von (3) die folgende Information über mich als Behauptender:

(5) Ich glaube, dass p.

In (1) steckt folglich insofern ein Widerspruch, als dass (1) aus (3) und (4) besteht, und die Behauptung von (3) die Information von (5) enthält. Wer also (1) behauptet, gibt damit zum Ausdruck, dass er p glaubt und nicht glaubt:

(6) Ich glaube, dass p und ich glaube nicht, dass $\mathrm{p}$.

Ein Widerspruch ist noch kein Paradox. Das eigentliche Paradox besteht darin, dass (1) zwar wahr sein kann, z.B. im Fall eines Irrtums, jedoch nicht aufrichtig und mit Bedacht behauptet werden kann, ohne etwas Widersprüchliches auszudrücken. Daraus folgt noch nichts über den Überzeugungsbegriff, denn Moores Diagnose begnügt sich mit dem Begriff der Behauptung. Weil die Behauptung, dass p, mit einschließt oder impliziert, dass der Behauptende von der Wahrheit dessen, was er behauptet, überzeugt ist, oder zumindest diesen Eindruck erwecken will, kann er die Wahrheit des Behaupteten nicht im gleichen Atemzug in Zweifel ziehen: „It is a paradox that it should be perfectly absurd to utter assertively words of which the meaning is something which may quite well be true - it is not a contradiction" (Moore 1993: 209). Der Widerspruch in (1) besteht zwischen dem durch die Behauptung Implizierten des ersten Konjunkts und dem zweiten Konjunkt. Paradox ist, dass der propositionale Gehalt einer Behauptung im Stil von (1) trotzdem wahr sein kann. 
3. Zur Verdeutlichung des Implikationsbegriffs mag jener Vergleich dienen, den Moore in seiner Besprechung von Russells Kennzeichentheorie anführt. Wir können die Bedeutung eines Satzes wie „Es gibt mindestens einen König von Frankreich“ nicht alternativ bestimmen, ohne von der herkömmlichen Bedeutung Gebrauch zu machen. "X' bedeutet nicht $\mathrm{X}$ " ist keine Kontradiktion im logischen Sinn, denn sonst wäre " $X$ ' bedeutet

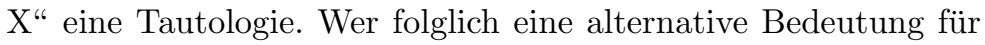
einen Ausdruck angibt, verstrickt sich nicht in einem Selbstwiderspruch. Und doch würde man mit der Behauptung ,'Es gibt einen König von Frankreich' bedeutet nicht, dass es einen König von Frankreich gibt" eine gewisse Absurdität zum Ausdruck bringen: „The absurdity I mean arises from the fact that when we use expressions to make an assertion, we imply by the mere fact of using them in accordance with established usage" (Moore 1944: 173). Behauptungen der Form "' $\mathrm{X}$ ' bedeutet nicht $\mathrm{X}$ “ sollen demnach dieselbe Absurdität aufweisen wie Behauptungen im Stil von (1), da die Angabe einer Bedeutungsalternative für "X“ ein Verständnis dessen voraussetzt, wovon die Alternative abweicht. Die implizierte herkömmliche Bedeutung steht im Widerspruch zur behaupteten Alternative.

Die folgende Begründung für die implizierte herkömmliche Bedeutung ist ein Beleg dafür, dass Moore die Tragweite seiner eigenen Argumentation unterschätzt. Mit dem Argument gegen die Möglichkeit, sich von der herkömmlichen Bedeutung sprachlicher Ausdrücke vollständig zu befreien, wäre Moore nahe daran, etwas über das Haben von Überzeugungen zu sagen. Ein Sprecher kann eine Behauptung nicht anders als vor dem Hintergrund seiner Überzeugungen aufstellen und sei es, um die Bedeutung der in der Behauptung enthaltenen Wörter zu verändern. Stattdessen begründet Moore das Zustandekommen der implizierten Bedeutung mit der empirischen Tatsache, dass in der Regel jemand auch glaubt, was er behauptet. „That we imply it means only, I think, something which results from the 
fact that people, in general, do not make a positive assertion, unless they do not believe that the opposite is true [...]. And it results from this general truth that a hearer who hears me say 'he has not gone out', will, in general, assume that I don't believe that he has gone out" (ibid.). Moores Implikationsbegriff beruht also auf dem Vertrauen, dass Behauptungen gewöhnlich nicht mit der Absicht zu lügen aufgestellt werden und daher bei den Zuhörern gerechtfertigt die Erwartung implizieren, dass der Sprecher der Überzeugung ist, dass das, was er sagt, wahr ist (Moore 1993: 210).

Selbstverständlich gibt es Gegenbeispiele und Wittgenstein dürfte es im Anschluss an Moores Vortrag nicht schwer gefallen sein, solche zu finden - das können wir indirekt aus einer Skizze mit Moores Antworten auf Wittgensteins Einwände schließen (ibid.: 207). Eine Person könnte etwa den Satz „I don't believe it's raining, but as a matter of fact it is" dann sinnvoll behaupten, wenn sie einer anderen Person einen bösen Streich spielen will und den zweiten Teil des Satzes einer weiteren Person leise ins Ohr flüstert, oder jemand könnte sich korrigieren wollen und, nachdem er die erste Hälfte des Satzes geäußert hat, einen Blick aus dem Fenster werfen und dann die zweite Hälfte des Satzes behaupten. Moores Reaktion auf Einwände dieser Art wirkt ad hoc: Sofern jemand mit gleichbleibender Lautstärke, ohne Unterbruch, ernsthaft und aufrichtig behauptet, dass er nicht glaube, dass es regnet, dass es aber regne, ergibt sich ein Widerspruch zwischen dem Implizierten und dem Behaupteten. Das Paradox würde einzig dann aufgehoben, wenn man in Behauptungen im Stil von (1) Tempus oder Person ändert (ibid.: 208).

Moores Erklärung für das Zustandekommen der Paradoxie weist jedoch einen weiteren Mangel auf. Moore begründet den Implikationsbegriff nicht nur mit dem natürlichen Verhalten einer Sprechermehrheit, sondern führt dieses auf die geistigen Zustände der Sprecher zurück (ibid.: 209). Wer behauptet, dass es regnet, 
soll sich in einem bestimmten Geisteszustand befinden, und dieser müsste sich ändern, wenn er aufrichtig behaupten wollte, dass er nicht glaube, dass es regnet. Diese Position dürfte Moore bereits in seinem Vortrag vertreten haben, denn Wittgenstein schreibt in einer Karte an Moore, in der er sich unter anderem für den Vortrag bedankt: To call this, as I think, you did, 'an absurdity for psychological reasons' seems to me to be wrong, or highly misleading“ (Wittgenstein 1974: 177). Wittgensteins Auseinandersetzung mit Moores Paradox richtet sich zu einem grossen Teil gegen die Identifikation von Überzeugungen mit geistigen Zuständen (PU, ii, x). Seine Argumentation wurde jüngst $\mathrm{zu}$ einer grundlegenden Kritik an funktionalistischen Theorien des menschlichen Geistes ausgearbeitet (Heal 1994).

4. Abgesehen vom strittigen Punkt, ob das Haben von Überzeugungen mit geistigen Zuständen gleichgesetzt werden kann, weist Moores Diagnose die Schwäche auf, dass die Anomalie auf die Erwartungen zurückgeführt wird, die ein Zuhörer einem Sprecher gegenüber hat, wenn dieser etwas behauptet. Schwach ist diese Erklärung deshalb, weil sie sich im Wesentlichen auf Sprechakte abstützt, ein Satz im Stil von (1) aber einen Gedanken ausdrückt, der unabhängig vom Sprechakt des Behauptens nicht gefasst werden kann. Schließlich hängt es nicht zwingend von einem Szenario mit Sprechern und Hörern ab, dass wir den Gedanken, der mit dem Satz „In diesem Zimmer brennt ein Feuer aber ich glaube es nicht" ausgedrückt wird, nicht richtig fassen können. Es ließe sich einwenden, dass man sehr wohl denken kann, dass in diesem Zimmer ein Feuer brennt, obwohl man es nicht glaubt, weil man bspw. das Feuer nicht bemerkt hat. Wer jedoch so argumentiert, nimmt stillschweigend einen Wechsel der Perspektive vor, denn der Gedanke, dass in diesem Zimmer ein Feuer brennt, wird sozusagen aus der Perspektive einer dritten Person und unabhängig vom Gedanken in der ersten Person, dass ich das Feuer nicht bemerke, gefasst. Und dieser Gedanke wäre eher mit dem Satz "Angenommen in diesem Zimmer brennt ein Feuer und ich bemerke es nicht" ausgedrückt und nicht 
mit „In diesem Zimmer brennt ein Feuer und ich glaube es nicht“.

Bernard Williams charakterisiert ein wesentliches Merkmal von Überzeugungen ${ }^{4}$ mit der Phrase „beliefs aim at truth“ (Williams 1970: 136). Damit meint er erstens, dass Wahr- und Falschheit die Bewertungsdimensionen von Überzeugungen sind. Wenn jemand etwas glaubt, dann ist seine Überzeugung wahr oder falsch. Zweitens wird jemand seine Überzeugung, dass p, aufgeben, wenn er erfährt, dass sie falsch ist, denn glauben, dass $\mathrm{p}$, ist dasselbe wie glauben, dass es wahr ist, dass p. Und drittens werden Überzeugungen in der Regel in Behauptungssätzen mitgeteilt, da eine Behauptung der Form "X glaubt, dass $\mathrm{p}$ “ im Allgemeinen mit sich bringt, dass $\mathrm{p}$ wahr ist (ibid.: 137). Überzeugungen können natürlich falsch sein, aber wir können uns nicht vornehmen, etwas Falsches zu glauben, wie wir uns etwa vornehmen können, in einem Spiel absichtlich zu verlieren, oder jemand durch eine falsche Behauptung in die Irre zu führen. Ein sicheres Indiz hierfür ist der Umstand, dass ein konstruiertes Verbum wie "falsch-glauben" (oder „flauben") in der ersten Person indikativ aktiv keine Verwendung findet. „Ich flaube, dass in diesem Zimmer ein Feuer brennt" weist dieselbe Absurdität auf, wie sie Moore bereits für sein Paradox diagnostiziert hat. Es ist zwar möglich, dass ich irrtümlich glaube, dass in diesem Zimmer ein Feuer brennt und den Raum fluchtartig verlasse, aber es wäre absurd, wenn ich von mir behaupten würde, dass ich flaube, dass in diesem Zimmer ein Feuer brennt.

Die Ähnlichkeit zu Moores Paradox ist offenkundig: So wie man nicht gleichzeitig behaupten kann, dass p, dass man aber nicht glaubt, dass $\mathrm{p}$, so kann man auch nicht glauben, dass $\mathrm{p}$, wenn man gleichzeitig der Überzeugung ist, dass $\mathrm{p}$ falsch ist. Glauben ist (wie Behaupten) wahrheitsgerichtet und es würde

${ }^{4}$ Wenn der Umstand, dass jemand etwas glaubt, nominalisiert werden soll, ziehe ich es vor, von „Überzeugungen" statt von "Glauben“ zu sprechen, da das englische Wortpaar „believe/belief“ keine elegante Entsprechung im Deutschen hat. 
einem Kunststück gleichkommen, sich diesem Merkmal von Überzeugungen entziehen zu können. „Perhaps, further, one objection to the projects of believing what is false is that there is no end to the amount you have to pull down. It is like a revolutionary movement trying to extirpate the last remains of the ancien régime" (ibid.: 151). Wird Moores Paradox ausschließlich im Rahmen von Sprechakten erklärt, bleibt also offen, weshalb es nicht möglich ist, den propositionalen Gehalt eines Satzes im Stil von (1) als Gegenstand einer Überzeugung zu haben. Verschiedene Autoren haben versucht, diese Lücke zu schliessen und stellen der Analyse Moore-paradoxer Sätze eine Analyse Moore-paradoxer Überzeugungen voran. Baldwin vertritt den Standpunkt, dass es dieselben Gründe sind, die sowohl das aufrichtige Behaupten Moore'scher Sätze als auch das Haben Moore'scher Überzeugungen verunmöglichen (vgl. Baldwin 1992: 230). Ich werde mich im Folgenden zwei Ansätzen zuwenden, die Moores Paradox auf der Ebene von Überzeugungen angehen, und versuche den Nachweis zu erbringen, dass es falsch ist, Moores Paradox in Bezug auf Behauptungen als davon abgeleitetes Problem aufzufassen. Daraus folgt nicht, dass sich in Bezug auf Überzeugungen kein Paradox ergibt, sondern dass die Richtung der Erklärung verkehrt ist. Auf diesen Punkt werde ich in den letzten beiden Teilen eingehen.

5. Shoemaker moniert, dass sprechakttheoretische Erklärungen von Moores Paradox nicht im Stande sind, zu erklären, weshalb der propositionale Gehalt eines Moore-paradoxen Satzes nicht geglaubt werden kann. "What really needs to be explained is why someone cannot coherently believe that it is raining and that she doesn't believe that it is, despite the fact that the conjuncts of this belief can both be true. If we can show that such beliefs are impossible [...] and if we can come up with an explanation of this, then an explanation of why one cannot (coherently) assert a Moore-paradoxical sentence will come along for free, via the principle that what can be (coherently) believed constrains 
what can be (coherently) asserted" (Shoemaker 1996: 76). ${ }^{5}$ Shoemakers Strategie scheint deswegen so attraktiv zu sein, weil sie zwei Fliegen auf einen Schlag zu erschlagen verspricht - gelingt es Moores Paradox in Bezug auf Überzeugungen zu erklären, erhält man eine Erklärung für die Paradoxie in Bezug auf Behauptungen kostenlos dazu.

Den Grund für die Verlagerung auf Überzeugungen sieht Shoemaker darin, dass der Inhalt einer Überzeugung im Stil von (1) selbstwidersprüchlich ist: „So it is a feature of the contents of Moore paradoxical sentences that if they can be believed at all, the subject of such a belief could not believe that she had it without believing a contradiction" (ibid.). Der Selbstwiderspruch ergibt sich entweder durch die Einschränkung auf bewusste Überzeugungen (Rosenthal 1995) oder durch eine weitere These die Shoemaker "self-intimation thesis" nennt (Shoemaker 1996: 80). Diese These soll erstens prinzipiell sicherstellen, dass Überzeugungen Überzeugungen zweiter Stufe nach sich ziehen: Wenn jemand glaubt, dass p, dann hat er auch die Überzeugung, dass er glaubt, dass p. Zweitens besagt die These, dass man den Überzeugungen, die man hat, mental zustimmt (,mental assent"). Überzeugungen zweiter Stufe, also Überzeugungen über Überzeugungen erster Stufe, sollen bei der Erklärung menschlichen Verhaltens eine zentrale Rolle spielen: „a rational person who believes that $\mathrm{p}$ will be disposed to behave in ways that indicate possession of the second order belief that she believes that p" (ibid.: 83). Es ist leicht ersichtlich, wie Shoemakers Prinzip einen Selbstwiderspruch in Moore-paradoxen Überzeugungen bewirkt. Wenn eine Person eine Überzeugung mit dem propositionalen Gehalt im Stil von (1) hätte, dann würde die Anwendung des Prinzips auf das erste Konjunkt zu einem Resultat führen (ich glaube, dass p), das dem zweiten

${ }^{5}$ Da es für den Hergang meiner Argumentation unerheblich ist, beziehe ich mich auf Shoemakers überarbeitete Fassung von Moore's Paradox and Self-knowledge (1995), wo einige Thesen in abgeschwächter Form vorgetragen werden. 
Konjunkt (ich glaube nicht, dass p) widerspricht (ibid.: 84).

Da dieses Argument in gewisser Weise auch nur für bewusste Moore-paradoxe Überzeugungen gilt - das sind Überzeugungen im Stil von (1), denen man mental zustimmt - bietet Shoemaker eine Variante des Arguments an, die auf den Begriff der mentalen Zustimmung verzichtet und Überzeugungen zweiter Stufe gänzlich in die menschliche Rationalität einzubetten versucht. Das Argument wird zuerst für Überzeugungen im Stil von (2) entwickelt und dann auf Überzeugungen im Stil von (1) übertragen. „First, if someone believes a proposition, she will be disposed to use that proposition as a premise in her reasonings, and second, if someone believes that she believes a proposition she will be disposed to use that proposition as a premise in her reasonings" (ibid.: 84-5). Analog dazu soll man, wenn man der Überzeugung ist, eine bestimmte Proposition nicht zu glauben, dazu disponiert sein, davon abzusehen, diese Proposition als Prämisse in rationale Überlegungen einzubeziehen (ibid.). Einen Moore-paradoxen Satz zu glauben, würde daher bedeuten, dass man dazu disponiert wäre, $\mathrm{p}$ als Prämisse in rationale Überlegungen einzubeziehen als auch davon abzusehen - und dies hält Shoemaker für unmöglich.

Kriegel kritisiert Shoemaker dafür, Moores Paradox auf eine konstitutive Beziehung zwischen Überzeugungen erster und zweiter Stufe zurückzuführen, da man nicht zwingend glauben muss, dass man etwas glaubt, wenn man etwas glaubt (Kriegel 2004: 107). Dennoch pflichtet Kriegel Shoemaker bei, dass Moores Paradox in Bezug auf Behauptungen auf eine logische Unverträglichkeit von Gedanken zurückzuführen ist: „It is not the subject's acts of asserting and believing that are absurd but also and primarily, what she asserts, or believes, that is absurd" (ibid.: 114). Anders als Shoemaker verfolgt Kriegel eine „two-step strategy“, derzufolge zuerst die Anomalie Moore'scher Behauptungen in Abhängigkeit von Moore'schen Überzeugungen erklärt werden soll, um dann unabhängig davon die Anomalie 
Moore'scher Überzeugungen erklären zu können (ibid.: 110).

Im Unterschied zu Shoemaker macht Kriegel ein Prinzip geltend, demzufolge Überzeugungen erster Stufe nicht von Überzeugungen zweiter Stufe begleitet werden, sondern diese als Teil enthalten. Wenn eine Person glaubt, dass p, dann hat sie eine aus zwei Konjunkten bestehende Überzeugung, dass p und dass sie glaubt, dass p, und nicht zwei Überzeugungen (ibid.: 108). Wendet man dieses Prinzip auf den Gehalt Moore-paradoxer Überzeugungen an, ergibt sich daraus bereits auf der Ebene des propositionalen Gehalts ein Selbstwiderspruch (ibid.: 109), und nicht wie bei Shoemaker erst dann, wenn eine Moore'sche Überzeugung als Prämisse in rationale Überlegungen einbezogen werden soll. Eine Moore'sche Überzeugung erweist sich nicht erst bei ihrer Einbindung als Prämisse in rationale Überlegungen als Hybrid aus zwei sich widersprechenden Überzeugungen, sie ist eine selbstwidersprüchliche Überzeugung. Moore-paradoxe Überzeugungen sind, so Kriegel, selbstwidersprüchlich, und dieser Selbstwiderspruch ist verantwortlich für die Anomalie Moore-paradoxer Behauptungen: „Moorean assertions are absurd because they express self-contradictory beliefs" (ibid.: 113). ${ }^{6}$

6. Wir sollten keinen Hehl daraus machen, dass sowohl Shoemakers als auch Kriegels Strategie voraussetzt, dass Behauptungen nichts Weiteres als geäußerte Überzeugungen sind. Beide Autoren messen dem Paradox in Bezug auf Überzeugungen eine explanatorische Priorität bei und rechnen damit, dass sich eine Erklärung für das Paradox in Bezug auf Behauptungen „for free“ ergibt. Kriegel nennt diesen Zugang „psychologism about Moore's para-

${ }^{6}$ Für Kriegel muss sich das Paradox in letzter Konsequenz sogar lösen lassen, wenn er den propositionalen Gehalt Moore-paradoxer Überzeugungen für ,semantically, rather than merely pragmatically, defective" hält (ibid.: 113). Wenn Moores Paradox auf eine Anomalie dessen, was geglaubt oder behauptet wird, zurückgeführt wird, dann kondensiert das Paradox zu einem grobschlächtigen Widerspruch und befreit von der paradoxen Situation, dass etwas nicht geglaubt oder behauptet werden kann, obwohl es möglicherweise wahr ist. 
dox" und plädiert dafür, dass sich die Anomalie von Moore'schen Überzeugungen auf Behauptungen vererbt: „It is Moorean beliefs that are fundamentally and inherently paradoxical, and Moorean assertions simply inherit their paradoxicality (if you please) from the Moorean beliefs they are used to express" (ibid.: 101, meine Hervorh.; vgl. 113). Shoemaker und Kriegel zufolge sind Behauptungen nichts anderes als laute Überzeugungen. ${ }^{7}$

Das Bild, das Shoemaker und Kriegel von Behauptungen zeichnen, erinnert stark an Freges Auffassung von Behauptungen: „Wir unterscheiden demnach 1. das Fassen des Gedankens - das Denken, 2. die Anerkennung der Wahrheit eines Gedankens - das Urteilen, 3. die Kundgebung des Urteils - das Behaupten" (Frege 1918: 62). Einer Behauptung gehen das Fassen und Beurteilen eines Gedankens voraus und das Behaupten besteht nur noch in der Veräußerlichung einer Prozedur, die im Inneren bereits stattgefunden hat. Die deutlichste Kritik an diesem Bild von Behauptungen stammt von Dummett (1973). Die Behandlung von Behauptungen als veräuäerlichte mentale Zustände oder Vorgänge vernachlässigt den konventionellen Aspekt, der für alle Sprechakte von zentraler Bedeutung ist. Dummett attestiert Frege, gesehen zu haben, dass die Theorie von Sinn und Bedeutung um eine Theorie des Behauptens, bzw. allgemein eine Theorie der Kraft, ergänzt werden muss, da die Wahrheitsbedingungen von Sätzen nicht offenbaren, was es heißt einen Satz zu behaupten - ,the point of doing so" (ibid.: 295). Dummett tadelt jedoch Frege, nie ganz von der Idee losgekommen zu sein, Behauptungen als sprachliche Veräußerlichung von etwas Innerem zu beschreiben (ibid.: 312). Ich bin der Auffassung, dass sich Dummetts Frege-Kritik generalisieren lässt und ein entscheidendes Defizit in Shoemakers

\footnotetext{
${ }^{7}$ Linville und Ring kritisieren dieses Verständnis von Behauptungen mit einem treffenden Bild: ,[B]elief is not, as traditionally assumed, the source of assertions (a mental reservoir, a neural network, or, whatever from which assertions flow); rather, belief is itself 'a new joint in', an elaboration or modification of, the concept of assertion" (Linville/Ring 1991: 306).
} 
und Kriegels Verständnis von Behauptungen aufweist. ${ }^{8}$

Der Umstand, dass bei Shoemaker und Kriegel vorwiegend von „Überzeugungen“, bei Frege bzw. Dummett jedoch von „Urteilen", „mental acts" oder „mental attitudes" die Rede ist, verlangt eine terminologische Vorbemerkung. Williams' Charakterisierung „, beliefs aim at truth“ trifft zumindest in Freges Verständnis auch auf Urteile zu. Ein Urteil zu fällen, heißt, die Wahrheit eines Gedankens anzuerkennen oder von einem Gedanken zu dessen Wahrheitswert „fortzuschreiten“ (Frege 1892: 34). Im Gegensatz zu Überzeugungen sind Urteile aber episodisch und nicht dispositional zu verstehen, da Urteile zumindest für Frege Taten und nicht Dispositionen sind. Der Begriff des Urteils betont, so könnte man sagen, den Akt des als wahr Anerkennens, während der Begriff der Überzeugung auf eine innere Bereitschaft zu diesem Akt abzielt. Es wird sich herausstellen, dass Moores Paradox in Bezug auf Überzeugungen inhärent mit dem episodischen Aspekt von Urteilen zusammenhängt. Für Dummetts Kritik am falschen Verständnis von Behauptungen, genügt es jedoch, Behauptungen als sprachliche Veräußerlichungen von etwas Mentalem zu interpretieren.

7. Gerade weil viele Sprechakte ein inneres Pendant zu haben scheinen (behaupten/urteilen, fragen/sich wundern etc.) liegt es nahe, Sprechakte durch die korrespondierenden Intentionen zu erklären. Der Versuch, Behauptungen allein in Rückgriff auf die Sprecherintentionen zu erklären, scheitert jedoch daran, dass Intentionen nicht zwingend festlegen, ob der Sprecher etwas behauptet; er könnte bspw. pathologisch lügen, eine Zeile aus einem

${ }^{8}$ Da Frege nicht namentlich am Sprechakt des Behauptens interessiert war und Behauptungen und Urteile hauptsächlich im Zusammenhang mit seinem logischen Schlussbegriff eine Rolle spielen, bedarf es einer eigenen Untersuchung, ob Dummetts Kritik gerechtfertigt ist. Im Fall von Shoemaker und Kriegel stellt sich die Situation anders dar, da diese Autoren für die Erklärung von Moores Paradox ein bestimmtes Verständnis von natursprachlichen Behauptungen voraussetzen. 
Gedicht zitieren oder eine Behauptung zum Zweck der Erörterung nur probeweise aufstellen. Der Erklärung anderer Sprechakte erweisen Intentionen ebenfalls einen Bärendienst - es ist bspw. denkbar, dass jemand etwas befiehlt, ohne die Absicht zu haben, dass der Befehl befolgt wird. Daraus folgt selbstverständlich nicht, dass den Sprecherabsichten keine explanatorische Relevanz zukommt, sondern dass Intentionen keinen geeigneten Ausgangspunkt für die Erklärung von Sprechakten darstellen. Dummett vertritt den Standpunkt, dass wir stattdessen bei den Konventionen ansetzen müssen, die mit dem jeweiligen Sprechakt verknüpft sind (Dummett 1973: 300f.).

Den Zusammenhang zwischen Sprechakt, Konvention und Intention erläutert Dummett (ibid.) anhand einer Analogie zu Spielen. So wie sich Spiele in Spiele mit und ohne Einsatz unterteilen lassen, so können Sprechakte in Sprechakte mit und ohne Konsequenzen unterteilt werden. Die Intentionen der Spieler bzw. der Sprecher fallen nur bei Spielen bzw. Sprechakten der zweiten Kategorie ins Gewicht. Das wird deutlich, wenn wir uns vorstellen, dass jemand ein Spiel verlieren will: Wer schlecht pokert, ob absichtlich oder nicht, verliert sein Geld, wer sich hingegen absichtlich in ein Matt manövriert, verliert zwar in gewisser Weise das Spiel, doch es ist fraglich, ob er überhaupt Schach gespielt hat. Wird diese Unterscheidung auf Sprechakte übertragen, entsprechen Befehle dem Pokern und Behauptungen dem Schach, da das Erteilen eines Befehls mit den Konsequenzen seines Nichtbefolgens verknüpft ist, das Aufstellen einer Behauptung hingegen keine derartigen Konsequenzen hat, sondern die Absicht des Sprechers zum Ausdruck bringt, etwas Wahres zu sagen: „Assertions take place against the background of a custom of uttering them with the intention of saying something true" (ibid.: 302).

Behauptungen sind zwar oft Ausdruck von Überzeugungen und Urteilen (ibid.: 330), aber Dummett warnt eindringlich davor, Überzeugungen und Urteilen in Bezug auf Behauptungen bloß die Rolle eines inneren Nexus beizumessen: „We have opposed 
throughout the view of assertion as the expression of an interior act of judgment; judgment, rather, is the interiorization of the external act of assertion. The reason for viewing the two this way round is that a conventional act can be described, without circularity, as the expression of a mental state or act only if there exist non-conventional ways of expressing it" (ibid.: 362). Die Umkehrung der Erklärungsrichtung wird mit folgendem Argument begründet: Eine Beschreibung von Behauptungen als veräußerlichte Urteile müsste den konventionellen Aspekt von Behauptungen auf der Ebene von Urteilen voraussetzen. Da es jedoch äußerst unklar ist, wie eine innere Version dieses konventionellen Aspekts lauten müsste, kann eine Handlung nur dann als Ausdruck einer mentalen Einstellung beschrieben werden, wenn wir der mentalen Einstellung auch anders Ausdruck verleihen können als mit einer Handlung, deren konventioneller Aspekt sich der rein mentalen Beschreibung entzieht. Im Gegensatz zur konventionellen Begrüßungsgeste als Ausdruck der Freude darüber, jemanden zu treffen, kennen wir für Urteile streng genommen keine alternativen Ausdrucksweisen zu sprachlichen Behauptungen. Also können Behauptungen nicht nur als Ausdruck von etwas Innerem beschrieben werden und es bleibt nur der Weg „von außen nach innen“ offen, das Urteilen durch das Behaupten zu erklären.

Leider lässt uns Dummett im Ungewissen, inwiefern die Auffassung von Behauptungen als Veräußerlichung mentaler Einstellungen zirkulär ist. Der Zirkel besteht vermutlich in der Unmöglichkeit, dass eine solche Beschreibung den konventionellen Aspekt von Behauptungen abdeckt, ohne auf Behauptungen als äußerliches Phänomen zu rekurrieren. Diesen Zirkel weist Dummett auch in einem etwas anderen Zusammenhang nach (ibid.: 298). Der konventionelle Aspekt von Behauptungen besteht darin, dass Behauptungen vor dem Hintergrund aufgestellt werden, dass der Sprecher die Absicht hat oder zu haben vorgibt, etwas Wahres zu äußern, und nicht bloß die, etwas zu verlauten, dessen Beurteilung er sich enthält. Das innere Pendant zu diesem Unterschied hat Frege mit der Unterscheidung zwischen dem bloßen Fassen 
eines Gedankens und dem Urteilen, dass der Gedanke wahr ist, zu beschreiben versucht: Das Urteilen verhält sich zum Fassen eines Gedankens wie das Behaupten zum bloßen Ausdrücken des entsprechenden Gedankens. Versucht man nun das innere Pendant dieser Unterscheidung einzig auf der Grundlage des Mentalen nachzuvollziehen, ohne auf das assertorische Moment von Behauptungen zu rekurrieren, tut sich ein schwer wiegendes Problem auf: Das bloße Fassen eines Gedankes weist in einem episodischen Sinn von "denken" nicht die gewünschte Neutralität bezüglich der Wahrheit des Gedankens auf, sondern eher Williams' Charakterisierung für Überzeugungen, auf Wahrheit abzuzielen. Der Unterschied zwischen dem bloßen Fassen eines Gedankens und dem Urteilen, dass er wahr ist, kollabiert in gewisser Weise, wenn man ihn aus der Perspektive der ersten Person zu beschreiben versucht und „denken" in einem episodischen Sinn, d.h. als ein von einer bestimmten Person zu einem bestimmten Zeitpunkt vollzogenen Akt, versteht.

8. Das Zusammenfallen von Urteilen und Denken in der ersten Person ist nicht nur ein starkes Argument für eine vom Behauptungsbegriff abgeleitete Bestimmungsweise des Urteilsbegriffs, sondern liefert auch den Schlüssel zu Moores Paradox in Bezug auf Überzeugungen. Shoemaker und Kriegel liegen richtig mit der Diagnose, dass die Anomalie von Sätzen im Stil von (1) nicht nur in Hinblick auf den Sprechakt des Behauptens erklärt werden darf. Der Grund für die Anomalie liegt aber weder in der Zurückführung von Überzeugungen der Form von (1) auf Dispositionen (Shoemaker) noch in der logischen Struktur des Gehalts von Überzeugungen dieser Form (Kriegel), sondern darin, was es für uns heißen würde, einen Gedanken dieser Form tatsächlich zu fassen. Es kann uns im episodischen Sinn von „denken“ nicht gelingen, zu denken, dass p der Fall ist, wenn dieselbe Episode von einem Akt geprägt ist, der in die gegenteilige Richtung zielt.

Mit dem, was ich als episodischen Aspekt des Fassens von Gedanken beschrieben habe, werden üblicherweise Urteile von Über- 
zeugungen abgegrenzt. Wenn sich daraus ergibt, dass sich das Denken zumindest unter bestimmten Gesichtspunkten in Urteilen vollzieht, dann muss dieses Resultat eingeschränkt werden. Der episodische Aspekt des Gedankenfassens ist selbstverständlich nicht so zu interpretieren, dass Gedanken qua gefasste wahr sind - schließlich liegen wir sehr oft falsch mit dem, was wir denken; es ist Gedanken wesentlich, dass sie auch falsch sein können. Wir dürfen nicht verkennen, dass die Formulierung, auf die Wahrheit „abzuzielen“, bloß teleologisch ist und in keiner Weise eine erkenntnistheoretischen Autorität zum Ausdruck bringt. Viel eher weist der episodische Aspekt des Gedankenfassens auf eine für das Denken konstitutive Autorität hin. ${ }^{9}$ Moores Paradox in Bezug auf Überzeugungen - das ist der Clou - beleuchtet etwas für das Denken Konstitutives, indem es auf einen Widerspruch hinweist, wenn etwas gedacht werden soll, das mit den Bedingungen für das Fassen von Gedanken konfligiert.

${ }^{9}$ Die Unterscheidung zwischen der epistemologischen und der konstitutiven Autorität des Fassens von Gedanken übernehme ich von Heal (1994: 21). 


\section{Literaturverzeichnis}

Baldwin, T.: G.E. Moore. London: Routledge, 1990.

Dummett, M.: Frege. Philosophy of Language. London: Duckworth, 1973, 2nd ed. Cambridge: Harvard University Press, 1981.

Frege, G.: Über Sinn und Bedeutung. In: Zeitschrift für Philosophie und philosophische Kritik 100, S. 25-50, 1892.

- Der Gedanke. In: Beiträge zur Philosophie des Deutschen Idealismus 2, S. 58-77, 1918.

Green, M./ Williams, J. N.: Moore's Paradox. New Essays on Belief, Rationality and the First Person. Oxford: Clarendon Press, 2007.

Kriegel, U.: Moore's Paradox and the Structure of Conscious Belief. In: Erkenntnis 61, S. 99-121, 2004.

Linville, K./ Ring, M.: Moore's Paradox Revisted. In: Synthese 87, S. 295-309, 1991.

Malcolm, N.: Ludwig Wittgenstein. A Memoir. London: Oxford University Press, 1994.

Moore, G. E.: A Reply to my Critics. In: Schilpp, P. (Hg.), The Philosophy of G.E. Moore, S. 535-677, Evanston: Northwestern University Press, 1942.

— Russell's "Theory of Descriptions". In: Schilpp, P. (Hg.), The Philosophy of Bertrand Russell, S. 175-225, La Salle: Open Court, 1944.

- Selected Writings. Hrsg. von T. Baldwin, London: Routledge, 1993.

Rosenthal, D. M.: Moore's Paradox and Consciousness. In: Philosophical Perspectives 9, S. 313-333, 1995.

Shoemaker, S.: Moore's Paradox and Self-knowledge. In: The First-Person Perspective and other Essays, S. 74-95, Cambridge: Cambridge University Press, 1996.

Williams, B.: Deciding to believe. In: Problems of the Self, S. 136-151, Cambridge: Cambridge University Press, 1973. 
Wittgenstein, L.: Letters to Russell, Keynes and Moore. Hrsg. von G. H. von Wright, Ithaca: Cornell University Press, 1974. Culture and Value. Hrsg. von G. H. von Wright. Übers. von P. Winch, Oxford: Blackwell, 1980.

Philosophisches Seminar der Universität Zürich Zürichbergstrasse 43

8044 Zürich

Schweiz 